\title{
Implementación de Lora y Lorawan como escenario futuro de la industrias 4.0 en el sector agroindustrial
}

peruano

Implementation of Lora of Lorawan as a future scenario of industry

4.0 in peruvian agro-industry sector

Recibido: noviembre 20 de 2019 | Revisado: diciembre 20 de 2019 | Aceptado: febrero 12 de 2020

\author{
DANIEl PÉREZ \\ REDY RISC ${ }^{2}$
}

\begin{abstract}
Resumen
El panorama actual del sector agroindustrial exige la inclusión de nuevas tecnologías que contribuyan a solucionar los constantes problemas a los que se enfrenta. Internet of Things se presenta como una alternativa para mitigarlos; sin embargo, las implementaciones de esta tecnología se realizan con el uso de redes de datos tradicionales como Wifi o redes celulares, las cuales representan un alto costo y consumo de energía. En esta investigación se realizó un análisis de la arquitectura y funcionamiento de la tecnología LoRa y el protocolo LoRaWAN, además de plantearse una serie de recomendaciones de cómo implementarla conjuntamente con otras tecnologías emergentes como es el BlockChain, todo esto, aplicado a la agroindustria peruana.
\end{abstract}

Palabras clave: Agroindustria, BlockChain, IoT, LPWAN, Perú

\begin{abstract}
The current panorama of the agribusiness sector, requires the inclusion of new technologies that contribute to solve the constant problems faced. Internet of Things is presented as an alternative to mitigate them, however, the implementations of this technology are developed with the use of traditional data networks such as Wifi or mobile networks, which represent a high cost and energy consumption. In this paper to make an analysis of the architecture and the operation of the LoRa technology and the LoRaWAN protocol, in addition to raising a series of recommendations of how to implement applications with other emerging technologies such as the BlockChain, all this, applied to Peruvian agribusiness.
\end{abstract}

Key words: Agro-industry, BlockChain, IoT, LPWAN, Peru
1 Facultad de Ingeniería, Universidad de Piura, Perú daniel.perez@posgrado.udep. edu.pe

(C) Los autores. Este artículo es publicado por la Revista Campus de la Facultad de Ingeniería y Arquitectura de la Universidad de San Martín de Porres. Este artículo se distribuye en los términos de la Licencia Creative Commons Atribución No-comercial - Compartir-Igual 4.0 Internacional (https://creativecommons.org/licenses/ CC-BY), que permite el uso no comercial, distribución y reproducción en cualquier medio siempre que la obra original sea debidamente citada. Para uso comercial contactar a: revistacampus@usmp.pe. 


\section{Introducción}

En el año 2020, aproximadamente, 50 mil millones de dispositivos se encontrarán conectados a Internet, esto debido al auge de tecnologías como el Internet de las Cosas o IoT (Mekki \& et al., 2019). Actualmente, el sector agrícola requiere automatizar gran parte de sus procesos, es por ello que el uso de soluciones de modernización, como el Internet de las cosas (IoT), cloud computing o big data se viene intensificando, todo esto con la finalidad de industrializar y mejorar sus sistemas productivos, debido al aumento en la demanda de alimentos y en su calidad (Tzounis \& et al., 2017). La finalidad es que los productores tengan la capacidad de usar esta tecnología y monitorear los sensores remotamente a través de una conexión a internet (Khanna \& et al., 2019).

Existen diversos problemas en la agricultura, los cuales podrían ser mitigados con el uso de IoT, además introducen el término "informática agrícola" o "agricultura electrónica", la cual busca contribuir en la mejora de la agricultura a través del uso de tecnologías de la información y comunicaciones o TIC (Mekki \& et al., 2015). Adicionalmente, el incremento de la población, sumado a los cambios climáticos, han provocado una disminución de las tierras cultivables $y$ recursos naturales, creando una preocupación real en la producción de alimentos de calidad a un menor costo, es aquí donde tecnologías como el IoT y el análisis de grandes cantidad datos, toman posicionamiento, usándose como herramienta para mejorar la productividad y eficiencia operativa en los procesos agrícolas (Elijah \& et al., 2018).
IoT y el uso de sensores inteligentes han venido demostrando claramente, su contribución en aplicaciones del sector agrícola. Para lograr ese cometido, se debe realizar un análisis exhaustivo de los requerimientos del negocio buscando que la tecnología pueda ser un medio de apoyo y no de obstaculización o demora en la mejora de los procesos productivos (Ray, 2017).

Kapoor \& et al. (2016) analiza a la denominada "agricultura inteligente" en la que el uso de sensores y actuadores son añadidos en las actividades diarias de la agroindustria. Además, señala que la agricultura de precisión, IoT, procesamiento de imágenes, telemetría, cloud computing, big data, inteligencia artificial, blockchain (Ferrández \& et al., 2016) son algunas de las tecnologías utilizadas actualmente, las cuales han venido teniendo un crecimiento exponencial en los últimos años (Stočes E et al., 2016).

Perú no es ajeno a la inclusión del IoT, es por ello que, diversos estudios, demuestran que la agroindustria peruana requiere el uso de tecnología para mejorar su producción tal como se aplica en la región de Piura, en donde se cuenta con un prototipo que incluye sensores para medir oxígeno, temperatura y dióxido de carbono en el proceso de fermentación de cacao, estos datos son mostrados en un sistema de información que ayuda a monitorear el proceso en tiempo real (Ipanaque \& et al., 2017).

Por otro lado, el uso de sensores para el control fitosanitario de las plantas de diversosfrutos, con lo cual se busca agregar inteligencia en los cultivos e identificar los problemas y aplicar medidas preventivas 
y correctivas únicamente a las zonas afectadas (Salas 2018). Los aspectos indicados, contribuyen a que tecnologías como las redes amplias de baja potencia (LPWAN) vayan tomando popularidad. Estas redes se están convirtiendo en uno de los pilares para la implementación de la Industria 4.0, siendo una alternativa frente a tecnologías tradicionales como 2G, 3G o 4G (Hernandez \& et al., 2017).

Petäjäjärvi \& et al. (2016) señala que entre las características más resaltantes de las redes LPWAN se encuentra su gran cobertura, bajo costo y consumo de energía, lo cual permite tener mediciones en tiempo real, además que operan en bandas de frecuencia sin licencia (sub $\mathrm{GHz}$ Industrial Scientific Medical ISM). Pierre Neumann (Neumann o et al., 2016) indica que, a diferencia de redes inalámbricas de corto alcance y de múltiples saltos, LPWAN permite lograr comunicaciones con un alcance mucho mayor y a una velocidad de bits baja, además que, entre las redes LPWAN, el protocolo LoRaWAN gana cada vez más interés por parte de las comunidades de investigación y el sector industrial.

Este protocolo asegura el establecimiento de comunicación por enlaces de largo alcance (en el orden de kilómetros) y está diseńado en una topología de red estrella donde cada nodo se comunica directamente con la estación base, lo cual representa una diferencia sustancial frente a otras redes, ya que permite que los dispositivos finales sean lo más simples y sencillos posibles, mientras que la complejidad de procesamiento es manejada por la estación base, con lo cual se consigue un bajo costo y consumo de energía, indicando que una batería portátil podría alcanzar una autonomía de varios años (Li \& et al., 2017). A pesar de lo mencionado anteriormente, en Perú se han realizado escasos estudios de LoRa (abreviatura de Long Range) y LoRaWAN, es por ello que, debido a las ventajas que traen inmersas, el presente estudio plantea el análisis de estas tecnologías en el sector agroindustrial peruano.

\section{Arquitectura}

El primer aspecto que se debe tomar en consideración es que LoRa es una tecnología de radiofrecuencia inalámbrica digital que proporciona el medio de conexión para el protocolo LoRaWAN, opera en las bandas de frecuencia de sub gigahercios y sin licencia (ISM). Las características clave de LoRa incluyen el bajo consumo de potencia y un rango de transmisión extremadamente largo, lo que hace que LoRa sea una opción para la conectividad LPWAN. El espectro extendido LoRa es una modulación patentada y desarrollada por SEMTEC, basada en la modulación del espectro extendido Chirp (CSS).

LoRa presenta también, ventajas sumamente resaltantes tales como la tolerancia a interferencias y la posibilidad de utilizar dispositivos como nodos donde los sensores son conectados y gateways que le posibilitan integrarse fácilmente con redes existentes y permite desarrollar aplicaciones de bajo coste para IoT (Rizzi \& et al., 2017).

En ese contexto, LoRaWAN, es un protocolo de comunicación para redes de área amplia y de baja potencia (LPWAN) diseñado para la conexión inalámbrica de dispositivos que utilicen baterías hacia la internet a través de redes 
regionales, nacionales o globales y que tengan una capacidad de comunicación bidireccional, segura de extremo a extremo, móvil y localizable.

La comunicación inalámbrica aprovecha las características de largo alcance de la capa física LoRa, lo que permite un enlace de un solo salto entre el dispositivo final y una o varias puertas de enlace (gateways). Todos los nodos son capaces de establecer comunicación bidireccional, así como multicast para grupos específicos con el fin de hacer un uso eficiente del espectro durante tareas tales como actualizaciones de firmware por aire u otros mensajes de distribución masiva (Feltrin \& et al., 2017).

Para resolver los requerimientos de una LPWAN existen otros protocolos de comunicación, en esta investigación se analizaron los que están introduciéndose en Perú, Sigfox y LoRaWAN. Tal como se observa en la Tabla 1, LoRaWAN presenta ventajas frente SigFox, siendo las más resaltantes, los niveles de seguridad que ofrece y la posibilidad de contar con un servicio gratuito, además de su bidireccionalidad (Mekki \& et al., 2017). Un detalle adicional en relación al tipo de redes se puede apreciar en la Figura 1.

Tabla 1

Comparativa: SigFox y LoraWAN

\begin{tabular}{|c|c|c|}
\hline Aspecto & SigFox & LoRaWAN \\
\hline Modulación & BPSK & CSS \\
\hline Frecuencia $(\mathrm{MHz})$ & \multicolumn{2}{|c|}{868 (Europa), 915 (América del Norte), 433 (Asia) } \\
\hline Ancho de banda & $100 \mathrm{~Hz}$ & $250 \mathrm{kHz}$ y $125 \mathrm{kHz}$ \\
\hline Velocidad de datos máxima & 100 bps & $50 \mathrm{kbps}$ \\
\hline Bidireccional & Limitado / Half-duplex & Sí / Semidúplex \\
\hline Máximo mensajes / día & $\begin{array}{l}140(\mathrm{UL}) \\
4(\mathrm{DL})\end{array}$ & Ilimitado \\
\hline Máxima carga útil (bytes) & $\begin{array}{l}12(\mathrm{UL}) \\
8 \text { bytes (DL) }\end{array}$ & 243 bytes \\
\hline Distancia & $10-40 \mathrm{~km}$ & $5-20 \mathrm{~km}$ \\
\hline Inmunidad a la interferencia & Muy alto & Muy alto \\
\hline Autenticación y encriptación & No & AES $128 b$ \\
\hline Servicio & Pagado & Gratuito / Pagado \\
\hline
\end{tabular}

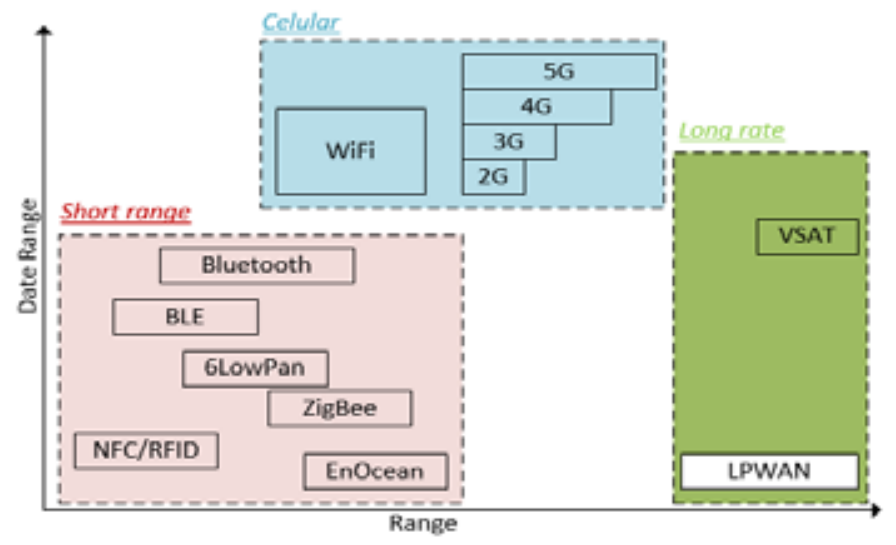

Figura 1. Tipo de redes 
En la Figura 2 se presenta una arquitectura típica de la red LoRa. En ella se observa la distribución de los dispositivos y cómo se conectan a una red en la nube.

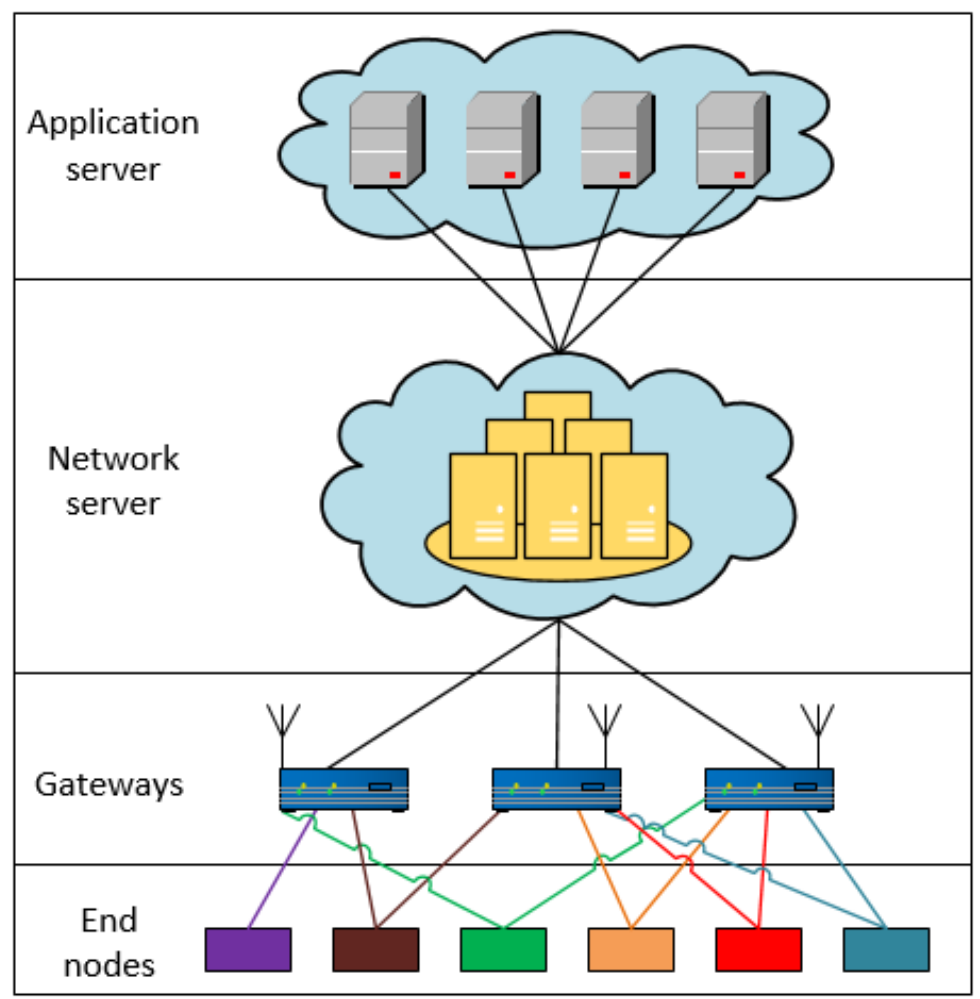

Figura 2. Red con tecnología LoRa

Un nodo final LoRa es un dispositivo de bajo consumo y gran potencia de transmisión que envía o recibe datos a un Gateway, cabe resaltar que se les puede dotar de comunicación entre sí. Es en el nodo final LoRa donde se conectan los sensores que conforman la Wireless Sensor Network (WSN) y son responsables de recoger los datos de campo (Khutsoane \& et al., 2017).

El Gateway es el dispositivo responsable de recibir la información del nodo y llevarla a la red; siendo la antena uno de sus elementos más importantes, ya que esta definirá el alcance de comunicación con los nodos finales. Según el tipo de gateway, pueden incluir desde 1000 nodos enviando información cada 30 segundos hasta
62500 nodos enviando información una vez al día (Navarro \& et al., 2018). LoRa Server es el servidor de red LoRaWAN, responsable de gestionar los registros duplicados, manejo de tramas recibidas de los gateways, tratamiento de la capa MAC de LoRaWAN y la programación de transmisiones de datos de enlace; LoRa server es considerado una de las partes clave de LoRa (Nor \& et al., 2017).

Finalmente, el Aplication server, es un elemento de tipo software responsable de visualizar la información enviada por los nodos (Feltrin \& et al., 2018). Es importante mencionar que LoRa trabaja en frecuencias sin licencia (ISM, Industrial, Scientific and Medical), las cuales están disponibles en todo el mundo, estas son $868 \mathrm{MHz}$ para Europa, 
$915 \mathrm{MHz}$ para América del Norte y 433 MHz para Asia (Aernouts \& et al., 2018). La Figura 3 muestra una representación de LoRa y el protocolo LoRaWAN, en el modelo OSI, en ella se reflejan las diferentes capas y su interacción desde las bandas en las que funciona, hasta llegar a la aplicación.

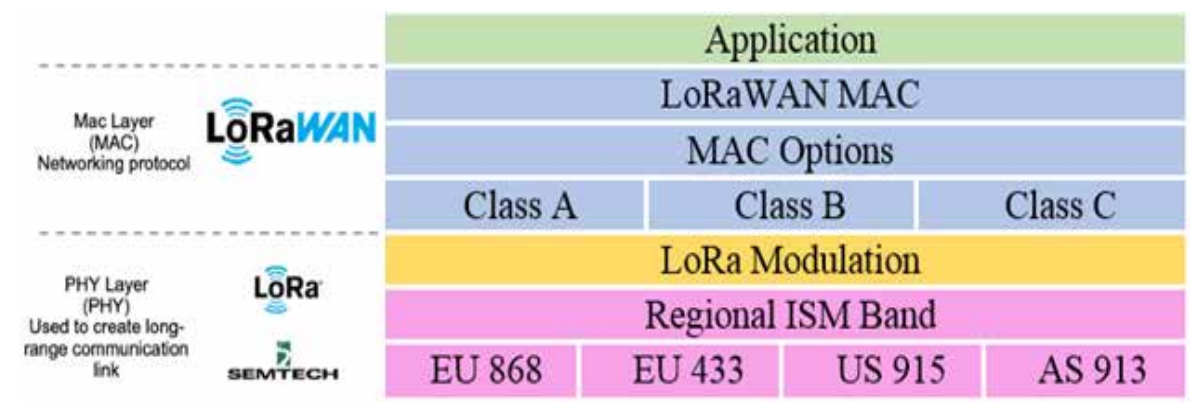

Figura 3. Arquitectura del protocolo LoRaWAN

LoRa utiliza la modulación de espectro expandido basada en la técnica chirp, la cual se encarga de modular el mensaje a través de una señal que cambia continuamente en frecuencia, logrando con esto, superar a DSSS (DirectSequence Spread Spectrum). LoRaWAN permite la conexión de tres clases de dispositivos según su estilo de comunicación (Sørensen \& et al., 2017): Clase A: Específica un punto final bidireccional que permite dos ventanas de recepción cortas después de cada transmisión hacia el Gateway, es ideal para dispositivos que usan una batería, pues requieren menor cantidad de energía para la operación. Clase B: Tienen ventanas de recepción programadas para recibir datos desde el Gateway; además de la capacidad de recepción de la Clase $\mathrm{A}$, los dispositivos clase $B$ permiten ventanas de recepción adicionales a una frecuencia sincronizada en el tiempo desde el gateway, pueden usar una batería dependiendo de los tiempos asignados de recepción. Clase C: Son continuamente receptivos a cualquier mensaje del gateway, por lo tanto, consumen la mayor cantidad de energía; su uso es recomendable cuando se cuenta con una fuente de alimentación externa.
Actualmente, los dispositivos de clase A son los que mejores prestaciones brindan a soluciones donde los sensores se encuentran ubicados en puntos sin acceso a fuentes de energía y por ende, sin acceso a otras redes de comunicación como los que se requerirían en los campos agrícolas del Perú; además, existen ya en el mercado nacional e internacional una gran cantidad de alternativas de bajo coste que pueden usarse para la creación de prototipos aplicados a necesidades concretas de datos e información.

\section{Funcionamiento}

Dentro de LoRaWAN, la agregación de nuevos nodos, es un punto sumamente importante, esta puede darse mediante comunicación punto-a-punto (P2P) en el que los dispositivos envían información directamente entre ellos, sin requerirse un elemento intermediario que administre la comunicación. Otra forma es con la denominada Mesh donde existe un nodo coordinador/ administrador de la comunicación entre ellos y la red; debido a que este solo puede escuchar a un nodo a la vez, la comunicación está limitada a 255 redes de 255 nodos (Tomasin \& et al., 2017). 
Las dos formas mencionadas anteriormente se encuentran dentro del denominado "modo LoRa" y presentan limitantes, principalmente, en conexiones a largas distancias y número de nodos. Esto se ve solucionado con el "modo LoRaWAN", en el que, para que un dispositivo final forme parte de la red, se requiere una serie de claves e identificadores del mismo. En el modo LoRaWAN, los nodos deben estar conectados a uno o varios gateways para poder unirse a la red. Esta red de nodos trabaja en una conexión tipo estrella, y es aquí donde entran a tallar las clases de nodos finales tratados en el apartado de Arquitectura (Yang \& et al., 2018).
En relación a los modos de conexión, existen dos. El modo OTAA (Over-TheAir-Activation) es considerado como el más seguro para conectarse a la red ya que la sesión se crea y renueva cada vez que el dispositivo pierde conexión, es apagado o reiniciado, dificultando que alguien pueda robar la sesión y adulterar el dispositivo. Los parámetros de configuración que utiliza son: DevEUI o identificador único de fábrica, AppEUI o identificador de aplicación único, AppKey o clave secreta de sesión AES 128 (Mohamed \& et al., 2018). El detalle del funcionamiento se observa en la Figura 4.

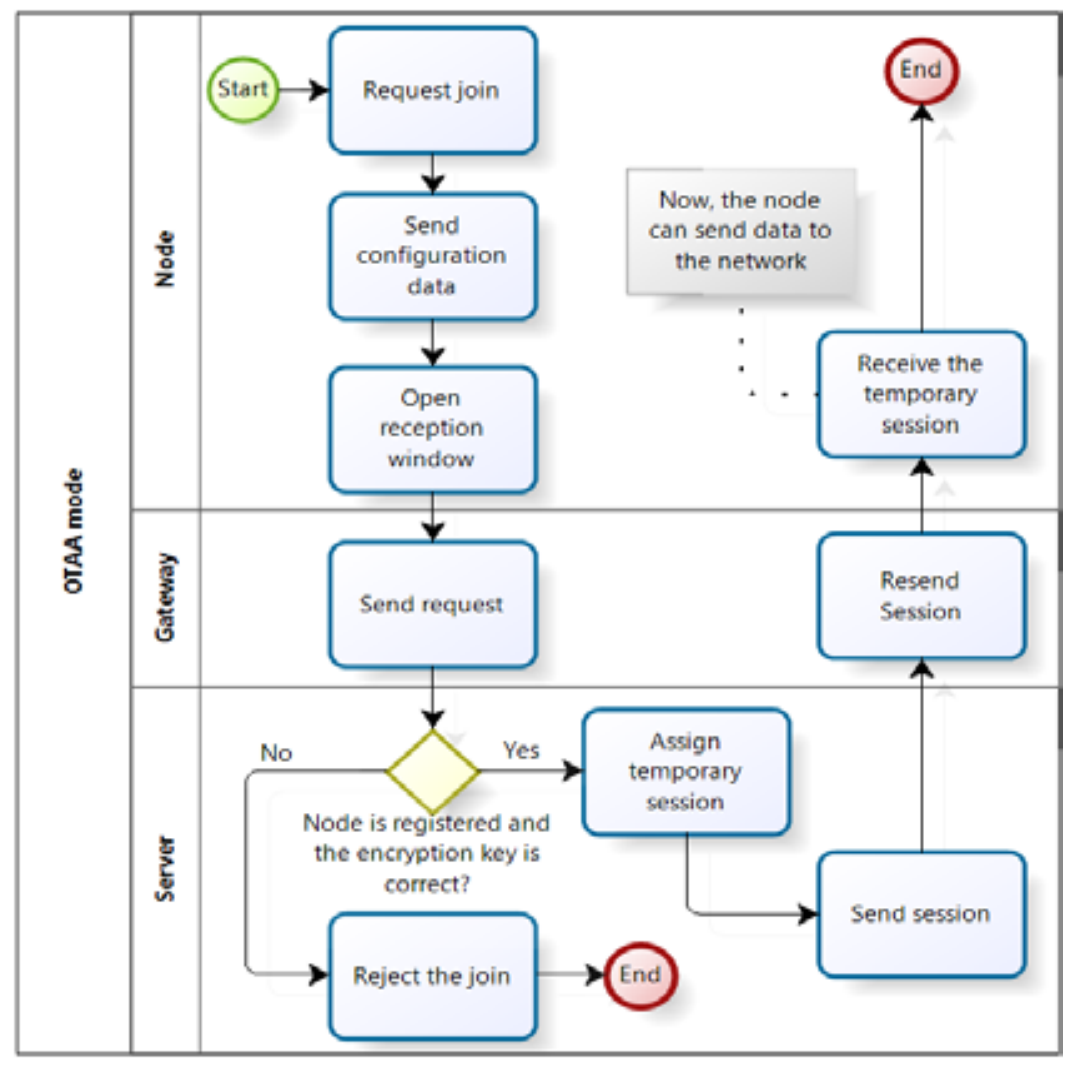

Figura 4. Modo OTAA

La Figura 5 muestra el funcionamiento del modo ABP (Activation By Personalization) en el que la sesión ya está asignada manualmente, por esa razón no se necesita solicitar un "join” ni que el servidor confirme los datos. Es por ello que si la clave de encriptación es encontrada podría ser adulterada y generar un peligro de seguridad. A pesar de ello, es bastante útil para nodos que 
no tengan una buena conexión o se encuentren en constante movimiento.

Los parámetros de configuración que utilizan son: DevAddress (dirección lógica utilizada para la comunicación con la red), NetworkSessionKey (clave cifrada entre el dispositivo y el operador para transmitir y validar la integridad de los mensajes) y ApplicationSessionKey (clave cifrada entre el dispositivo y el operador mediante la aplicación), utilizada para fines similares a los del NetworkSessionKey (Gresak \& et al., 2019). Sin embargo, el modo OTAA es el más utilizado ya que se considera más seguro.

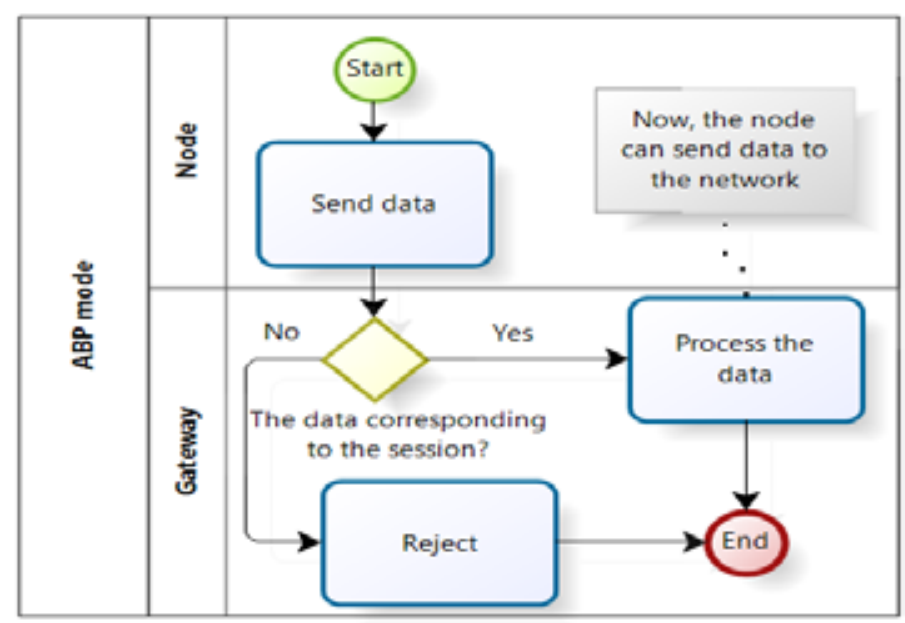

Figura 5. Modo ABP

En relación a los nodos finales, estos recopilan datos de los sensores, los transmiten y reciben información desde el Gateway, el cual actúa como un puente transparente y retransmite los datos bidireccionales entre los nodos finales y el servidor de red. El servidor de red se conecta a uno o muchos gateways ya sea por conexión cableada o de manera inalámbrica, verifica todos los aspectos relacionados a seguridad, gestiona los nodos finales y la duplicidad de mensajes; teniendo en cuenta, que cuanta mayor sea la frecuencia de envío y recepción de mensajes, la vida útil de la batería del nodo final, disminuirá.

Finalmente, el servidor de aplicación colecciona y analiza los datos de los nodos finales; es aquí donde se pueden desarrollar aplicaciones IoT para tratamiento y visualización de los datos que generan, además de aplicaciones de inteligencia artificial y análisis de datos para obtener información útil en la toma de decisiones.

En resumen, los datos (mediciones tomadas desde el campo) son transmitidos por nodos finales hacia los gateways, estos los gestionan y envían hacia un servidor de red; el servidor gestiona los paquetes duplicados, ejecuta comprobaciones de seguridad y administra la red, finalmente, los datos se reenvían a los servidores de aplicaciones para su tratamiento.

\section{Análisis}

Perú es uno de los protagonistas de la industria alimenticia global con productos como el cacao, espárrago, palta, quinua, arándano, uva, papa, algodón, entre otros. La agroindustria es considerada como uno de los pilares 
en la economía peruana, siendo este uno de los sectores exportadores que más ha crecido en los últimos años; sin embargo, de las más de 5 millones de parcelas agrícolas, el $79 \%$ presenta un tamaño menor a las cinco hectáreas; así mismo, del $100 \%$ de empresas en el sector, el $95 \%$ es considerada como pequeña o mediana, existiendo una gran cantidad de áreas agrícolas con ingresos negativos (Figura 6 y Tabla 2).

Esto dificulta la introducción de nuevas tecnologías que eleven el rendimiento y productividad de los campos (Escobar, 2015).

Tabla 2

Unidades agricolas peruanas (AU), parcelas y área promedio

\begin{tabular}{llll}
\hline \multicolumn{1}{c}{ Tamaño $(\mathrm{Ha})$} & \multicolumn{1}{c}{ Número AU } & \multicolumn{1}{c}{ Nro. de parcelas } & \multicolumn{1}{c}{ Área $(\mathrm{Ha})$} \\
\hline Menor que 0.5 & 507137 & 1098810 & 99700 \\
Entre 0.5 y 2.9 & 996277 & 2389388 & 1272793 \\
Entre 3 y 4.9 & 251001 & 648929 & 896259 \\
Entre 5 y 9.9 & 218564 & 546384 & 1418311 \\
Entre 10 y 19.9 & 118274 & 270775 & 1522078 \\
Entre 20 y 49.9 & 75435 & 148997 & 2172245 \\
Entre 50 y 99.9 & 23363 & 44240 & 1519797 \\
Mayor que 100 & 23455 & 44132 & 29841281 \\
\hline
\end{tabular}

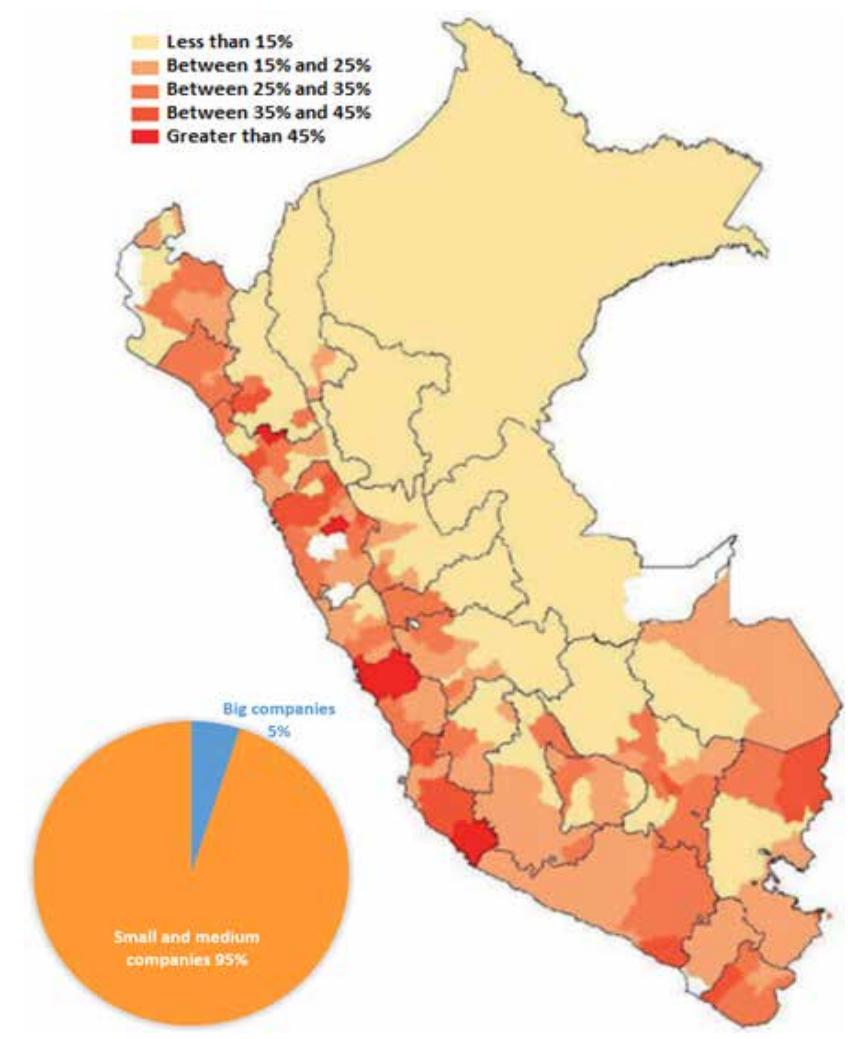

Figura 6. Provincias peruanas con alta incidencia de unidades agrícolas e ingreso agrícola neto negativo, y unidades agrícolas en pequeñas, medianas y grandes empresas 
Sigue siendo un gran desafío para desarrollar una agricultura de precisión, no contar con infraestructura de red de comunicación en zonas remotas, además que, debido a lo expuesto en el párrafo anterior, muchos agricultores no pueden asumir los costos para la compra de dispositivos IoT pues los costos que incurren, le puede resultar relativamente altos. Los beneficios de la agricultura de precisión o agricultura inteligente mediante el uso de la IoT, permiten la obtención de un doble beneficio; en primer lugar, ayuda a reducir los costos de producción y disminuye los desperdicios de insumos (el agua fundamentalmente) $y$, además permite incrementar el rendimiento al facilitar la toma de decisiones oportuna basada en datos más precisos.

A la fecha, existe documentación relacionada a implementaciones o investigaciones de IoT en el sector agroindustrial peruano, las cuales fueron desarrolladas con tecnologías tradicionales; estas presentan una serie de desventajas como son la baja autonomía de la batería, altos costes y poca distancia de envío de los datos capturados por los sensores, además de requerir un constante monitoreo y mantenimiento in situ, incrementado así los gastos operativos.

Se ha realizado una búsqueda exhaustiva de la aplicación de LoRa y LoRaWAN en la agroindustria peruana, sin haber encontrado información formal, a pesar que el Perú es un país en el que el 35\% de la innovación se viene promoviendo en ese sector y que el agro es la segunda fuente generadora de divisas. Creemos que una posible causa sea el déficit de estudios que muestren correctamente las ventajas que trae consigo la tecnología, existiendo muchos casos de éxito en otros países como por ejemplo España (Borrero \& et al., 2019).

\section{Propuesta}

Una alternativa de solución ante los problemas señalados es el desarrollo de proyectos piloto que incluyan el uso de una red de sensores inalámbricos IoT con tecnologíaLoRayel protocoloLoRaWAN; estas plantas deben ser desarrolladas con el apoyo de organizaciones de agricultores denominadas "comisiones de regantes" y juntas de usuarios. Se proponen los siguientes pasos: (i) Determinar el área cultivada por la organización. (ii) Identificar los cultivos con más baja producción y rendimiento. (iii) Determinar los parámetros a medir que representen mayor influencia en la producción y rendimiento: condiciones climatológicas, datos agronómicos, consumo hídrico, etc. (iv) Realizar la selección de los sensores y nodos LoRa adecuados. (v) Seleccionar y ubicar los gateways en las zonas de las comisiones con disponibilidad de energía y acceso a internet. (vi) Subir la data e información a servidores en la nube. (vii) Desarrollar aplicaciones web necesarias para el acceso a la información por parte de los agricultores. (viii) Evaluar los resultados al final de la campaña agrícola.

En relación a la data recolectada por los sensores, esta deberá transformarse en información en una plataforma de internet of things, la cual, mediante la utilización de tecnologías como big data, cloud computing, inteligencia artificial, realidad aumentada, ciberseguridad, proporcione criterios para que los pequeños agricultores tomen decisiones 
con mayor precisión y en el momento oportuno.

Por otro lado, debido a las grandes distancias de cobertura inalámbrica de LoRaWAN, su bajo costo y consumo de batería, además del reducido tamaño que presentan los dispositivos LoRa, cumplirían con los requerimientos para monitorear las condiciones climáticas como radiación solar, humedad, lluvia, entre otros; los cuales complementen los datosoficiales deestaciones meteorológicas, especialmente en períodos como los del fenómeno del Niño, pero que estén al alcance de los pequeños agricultores. Esta red de sensores y la información generada, puede ser utilizada como mecanismo de reducción de costos logísticos y como medida de impulso a la competitividad a través del seguimiento que se le realice a los alimentos cuando estos son transportados, agregando un eslabón de calidad adicional el en ciclo de vida del producto. Teniendo en cuenta que los parámetros medidos que normalmente requiere el sector agrícola varían muy lentamente, que la data que se obtiene genera un tamaño reducido en el paquete de envío de datos y que en los cultivos agrícolas se deben cubrir áreas extensas, LoRa y LoRaWAN representan una excelente alternativa para resolver esta problemática.

Implementada la red de datos, el paso siguiente es desarrollar las interfaces (a nivel de aplicaciones web) para que los agricultores, operadores y organismos implicados tengan la información que necesitan para la toma de decisiones, buscando que todos los stakeholders cuenten con una solución tecnológica que les permita gestionar eficientemente y en tiempo real los cultivos e insumos necesarios.
En relación a temas de seguridad, aunque LoRa y LoRaWAN son opensource, y en su modo de conexión OTAA ofrecen el estándar de cifrado avanzado AES 128; se recomienda la inclusión de blockchain, tecnología que recolecta información en un sistema transparente y colaborativo entre todos sus miembros (Cortez \& et al., 2019), lo que incrementa el nivel de seguridad al garantizar la transparencia e inmutabilidad de cada transacción debido a que los datos no pueden ser alterados por elementos externos a la red (Lin \& et al., 2017). Esto se aplica al momento de ańadir nuevos nodos finales y en el proceso de envío de datos hacia los servidores de aplicaciones.

\section{Conclusiones}

El sector agroindustrial peruano ha presentado un crecimiento constante en los últimos años, tanto en áreas cultivadas o niveles de exportación; sin embargo, para el pequeño y mediano agricultor, el cual representa más del $80 \%$, la producción, el rendimiento y los resultados no son los mejores. Es por ello que, es sumamente importante desarrollar soluciones tecnológicas de bajo coste que permitan recolectar data de estos cultivos. En la Agricultura inteligente, el uso de sensores $\mathrm{y}$ aplicaciones $\mathrm{IoT}$, requiere que los datos sean monitoreados remotamente y los gastos operativos sean reducidos al máximo. LoRa es una tecnología de bajo costo y fácil de implementar, la cual permite conectar distintos tipos de sensores en distancias extensas de terreno, además que, debido a su largo alcance y bajo consumo, reduce costos operativos, de mantenimiento, infraestructura o reemplazo de baterías. En la agroindustria peruana, se suelen medir 
aspectos como temperatura, humedad, $\mathrm{PH}$ del suelo entre otros, data que no cambia su valor en intervalos de tiempo pequeños, además que estas mediciones y su correspondiente envío, representan poco espacio de almacenamiento $y$ transmisión. Generalmente los sensores deben ser instalados en zonas extensas de terreno, por lo que es sumamente necesario contar con dispositivos que incluyan autonomía energética. Para otros requerimientos que no se ajusten a estas especificaciones como por ejemplo enviar imágenes o videos, LoRaWAN no debería tomarse como una alternativa pues no brindará buenos resultados. Finalmente, el uso de estas soluciones tecnológicas, servirá de ayuda al agricultor como herramienta de monitoreo remoto de cultivos, y planteará una base para la implementación técnicas como el riego inteligente, control fitosanitario, análisis de suelo y microclima, pronósticos de producción, entre otros, y por lo tanto traerán consigo un incremento en la producción agroindustrial.

\section{Referencias}

Aernouts, M.; Berkvens, R.; Van Vlaenderen, K.; Weyn, M. (2018) Sigfox and LoRaWAN Datasets for Fingerprint Localization in Large Urban and Rural Areas. Data, 3, 13. https://doi.org/10.3390/ data3020013

Borrero J.; Fernández G.; Rodríguez C. (2018). Aplicación de la tecnología LoRaWAN en la agricultura. Bo true activities, h, pp. 1-8. https://doi.org/10.13140/ RG.2.2.24492.77443

Cortez-Zaga C.; Casas-Llamacponcca V.; Shiguihara P. (2019). Technological model for the exchange of goods in the Peruvian agricultural business sector using the smart contracts and blockchain. Smart Innovation, Systems and Technologies, vol. 140, pp. 585-593. https://doi. org/10.1007/978-3-030-16053$1 \_57$
Dlodlo N.; Kalezhi J. (2015). The internet of things in agriculture for sustainable rural development. International Conference on Emerging Trends in Networks and Computer Communications, pp. 13-18. https://doi.org/10.1109/ ETNCC.2015.7184801

Elijah O.; Rahman T.; Orikumhi I.; Leow C.; Hindia M. (2018). An Overview of Internet of Things (IoT) and Data Analytics in Agriculture: Benefits and Challenges. IEEE Internet Things Journal, vol. 5, no. 5, pp. 3758-3773. https://doi. org/10.1109/JIOT.2018.2844296

Escobar A. (2015). Agricultura peruana: nuevas miradas desde el censo agropecuario. Red de bibliotecas virtuales de ciencias sociales de américalatinayelcaribe. Recuperado de http://biblioteca.clacso.edu. ar/Peru/grade/20160217082754/ LIBROGRADE_CENAGRO.pdf 
Feltrin L.; Buratti C.; Vinciarelli E.; De Bonis R. and Verdone R. (2018) LoRaWAN: Evaluation ofLink-and System-Level Performance. IEEE Internet of Things Journal, vol. 5, no. 3, pp. 2249-2258. https://doi. org/10.1109/JIOT.2018.2828867

Feltrin L.; Buratti C.; Vinciarelli E.; De Bonis R.; Verdone R. (2018). LoRaWAN: Evaluation ofLink-and System-Level Performance. IEEE Internet of Things Journal, vol. 5, no. 3, pp. 2249-2258. https://doi. org/10.1109/JIOT.2018.2828867

Ferrández-Pastor F.; García-Chamizo J.; Nieto-Hidalgo M.; MoraPascual J.; Mora-Martínez J. (2016). Developing ubiquitous sensor network platform using internet of things: Application in precision agricultura. Sensors (Switzerland), vol. 16, no. https:// doi.org/10.3390/s16071141

Gresak E.; Voznak M. (2019) Protecting Gateway from ABP Replay Attack on LoRaWAN. International Conference on Advanced Engineering Theory and Applications. V. 554. Springer. https://doi.org/10.1007/978-3030-14907-9_39

Hernandez D.; Peralta G.; Manero L.; Gomez R.; Bilbao J.; Zubia C. (2017). Energy and coverage study of LPWAN schemes for Industry 4.0. IEEE International Workshop of Electronics, Control, Measurement Signals and their Application to Mechatronics. h t t p s://doi.org/10.1109/ ECMSM.2017.7945893
Ipanaque W.; Belupu I.; Castillo J.; Salazar J. (2017). Internet of things applied to monitoring fermentation process of cocoa at the Piura's mountain range. Chilean Conference on Electrical, Electronics Engineering, Information and Communication Technologies, pp. 1-5. https://doi.org/10.1109/ CHILECON.2017.8229532

Kapoor A.; Bhat S.; Shidnal S.; Mehra A. (2016). Implementation of IoT (Internet of Things) and Image processing in smart agricultura. International Conference on ComputationSystem and Information Technology for Sustainable Solutions, pp. 21-26. https://doi.org/10.1109/ CSITSS.2016.7779434

Khanna A.; Kaur S. (2019). Evolution of Internet of Things (IoT) and its significant impact in the field of Precision Agriculture. Computers and Electronics in Agriculture, vol. 157. Elsevier B.V., pp. 218231. https://doi.org/10.1016/j. compag.2018.12.039

Khutsoane O.; Isong B.; Abu-Mahfouz A. (2017) IoT Devices and Applications based on LoRa/ LoRaWAN. Annual Conference of the IEEE Industrial Electronics Society, Beijing, pp. 61076112. https://doi.org/10.1109/ IECON.2017.8217061

Li L.; Ren J.; Zhu Q. (2017). On the application of LoRa LPWAN technology in Sailing Monitoring System. Annual Conference on Wireless On-Demand Network Systems and Services 
2017 - Proceedings, pp. 77-

80. https://doi.org/10.1109/

WONS.2017.7888762

Lin J.; Shen Z.; Miao C. (2017). Using blockchain technology to build trust in sharing LoRaWAN IoT. ACM International Conference Proceeding Series, vol. Part F1306, pp. 38-43. https://doi. org/10.1145/3126973.3126980

Mekki K.; Bajic E.; Chaxel F.; Meyer F. (2018). Overview of Cellular LPWAN Technologies for IoT Deployment: Sigfox, LoRaWAN, and NB-IoT. IEEE International ConferenceonPervasiveComputing and Communications Workshops (PerCom Workshops), Athens, pp. 197-202. https://doi.org/10.1109/ PERCOMW.2018.8480255

Mekki K.; Bajic E.; Chaxel F.; Meyer F. (2019). A comparative study of LPWAN technologies for largescale IoT deployment. ICT Express, vol. 5, no. 1, pp. 1-7. https://doi. org/10.1016/j.icte.2017.12.005

Mohamed E.; Butun I.; Pereira N.; Gidlund M. (2018). Formal security analysis of LoRaWAN. Computer Networks v. 148, p. 328339. https://doi.org/10.1016/j. comnet.2018.11.017

Navarro J.; Sendra S.; Ameigeiras P.; Lopez J. (2018). Integration of LoRaWAN and 4G/5G for the Industrial Internet of Things. IEEE Communications Magazine, vol. 56, no. 2, pp. 6067. https://doi.org/10.1109/ MCOM.2018.1700625
Neumann P.; Montavont J.; Noel T (2016). Indoor deployment of low-power wide area networks (LPWAN): A LoRaWAN case study. International Conference on Wireless and Mobile Computing, Networking and Communications. https://doi.org/10.1109/ WiMOB.2016.7763213

Nor R.; Zaman F.; Mubdi S. (2017) Smart traffic light for congestion monitoring using LoRaWAN. IEEE 8th Control and System Graduate Research Colloquium (ICSGRC), Shah Alam, 2017, pp. 132-137. https://doi.org/10.1109/ ICSGRC.2017.8070582

Petäjäjärvi J.; Mikhaylov K.; Hämäläinen M.; Iinatti J. (2016). Evaluation of LoRa LPWAN technology Evaluation of LoRa LPWAN Technology for Remote Health and Wellbeing Monitoring. Med. Inf. Commun. Technol. (ISMICT) 10th Int. Symp. on. IEEE, pp. 1-5. https://doi.org/10.1109/ ISMICT.2016.7498898

Ray P. (2017). Internet of things for smart agriculture: Technologies, practices and future direction. J. Ambient Intell. Smart Environ. vol. 9, no. 4, pp. 395-420. https:// doi.org/10.3233/AIS-170440

Rizzi M.; Ferrari P.; Flammini A. (2017). Evaluation of the IoT LoRaWAN Solution for Distributed Measurement Applications. IEEE Transactions on Instrumentation and Measurement, vol. 66, no. 12, pp. 3340-3349. https://doi. org/10.1109/TIM.2017.2746378 
Salas E. (2018). IoT para Automatizar red de trampas de moscas de la fruta, pp. 1-10. https://www.researchgate. net/publication/327830232_ IoT_para_Automatizar_red_de_ trampas_de_moscas_de_la_fruta

Sørensen R.; Kim d.; Nielsen J.; Popovski P. (2017). Analysis of Latency and MAC-Layer Performance for Class A LoRaWAN. IEEE Wireless Communications Letters, vol. 6, no. 5, pp. 566-569. https://doi. org/10.1109/LWC.2017.2716932

Stočes M.; Vaněk J.; Masner J.; Pavlík J. (2016). Internet of things (IoT) in agriculture - Selected aspects. Agris On-line Pap. Econ. Informatics, vol. 8, no. 1, pp. 83-88. https:// doi.org/10.7160/aol.2016.080108

Tomasin S.; Zulian S.; Vangelista L. (2017). Security Analysis of LoRaWAN Join Procedure for Internet of
Things Networks. IEEE Wireless Communications and Networking Conference Workshops (WCNCW), San Francisco, CA, 2017, pp. 1-6. https://doi.org/10.1109/ WCNCW.2017.7919091

Tzounis A.; Katsoulas N.; Bartzanas T.; Kittas C. (2017). Internet of Things in agriculture, recent advances and future challenges". Biosystems Engineering, vol. 164. Academic Press, pp. 3148. https://doi.org/10.1016/j. biosystemseng.2017.09.007

Yang X.; Karampatzakis E.; Doerr C.; Kuipers F. (2018). Security Vulnerabilities in LoRaWAN. IEEE/ACM Third International Conference on Internet-of-Things Design and Implementation (IoTDI), Orlando, FL, pp. 129140. https://doi.org/10.1109/ IoTDI.2018.00022 
